\title{
ABSTRACTS
}

\section{I.-PITUITARY AND CHIASMA}

(I) Christiansen, V. (Copenhagen). - Concerning supra-sellar tumours. (A propos des Tumeurs Supra-sellaires). Arch. d'Ophtal., October, 1929.

(1) Although of much interest to ophthalmologists this comprehensive paper extending to 34 pages, is addressed more directly to neurologists. In this abstract the wording of the original is followed as closely as possible.

Topographical delimitation of the supra-sellar region, even approximately, is impossible. Physiological experiments in that direction although of great interest, do not agree fully with our clinical knowledge and technique of examination. From the clinical point of view, grouping supra-sellar tumours as a nosological entity is chimerical. In many cases we are fortunate if we succeed in diagnosing a tumour in the chiasmal region without more exact localisation.

Chiasmal symptoms alone enable us, though not invariably, to localise a lesion in this region of the base. To determine a supraor intra-sellar seat of tumour is in the majority of instances beyond the skill of the clinician. Nutritional phenomena, e.g., adiposity, anhydrosis, amenorrhoea, atrophy or precocious development of the genital organs, are present or are lacking, independently of the intra- or extra-sellar site of the growth. Numerous other symptoms such as polyuria, abnormal pigmentation, etc., occur with equal frequency in supra-sellar and intra-sellar tumours. In discussing cerebral tumours, whatever their localisation, the question is nearly always one of large tumours, whether giving rise to unilateral or bilateral symptoms. A comparison of the results obtained by the physiologist and by the clinician is invariably disconcerting. The physiologist is able to destroy a strictly limited area of the brain substance and to study, under ideal conditions, the results of his intervention. But the medical clinic imitates physiological experiments maladroitly. The symptoms caused by tumours in the chiașmal region are much more complicated and less easy to unravel.

It is common knowledge that the adiposo-genital syndrome is induced by a tumour situated in the interior of the hypophysis. Probably this syndrome follows destruction by compression or invasion of basal centres, but anatomical and clinical proof is very difficult by reason of the dimensions of the tumour. The clinician is familiar with the adiposo-genital syndrome resulting 
from a supra-sellar tumour, but in view of the size of the growth it is almost impossible to exclude a direct action upon the pituitary gland.

There is, however, a clinical distinction between supra- and intra-sellar tumours. Until recently when acromegaly and adiposo-genital dystrophy were described, we had practically exhausted the clinical knowledge of tumours in this region. But now we all know that in the majority of cases of tumour in the chiasmal area, endocrine symptoms are lacking. The symptoms which dominate the clinical features of this region are simple chiasmal symptoms, i.e., affection of the basal optic paths, alterations in the sella turcica or other parts of the bones of the base of the skull, and cytological changes in the cerebro-spinal fluid. Occasionally, in addition, there are indications of partial hypophyseal dysfunction. Among more than 60 cases of tumour in the chiasmal region, less than one third showed hypophyseal symptoms even if rudimentary symptoms were included : the great majority exhibited simple chiasmal symptoms. The majority of tumours in the chiasmal region present supra-sellar symptoms although not in the common acceptance of the term; the basal ganglia are not affected but the basal optic paths, also placed above the sella turcica, rarely escape involvement.

In the evolution of an intra-sellar tumour we sometimes find hypophyseal symptoms combined with those of the adiposo-genital type. But in all such cases under observation the basal optic paths, intermediate between the hypophysis and the quadrigeminal tubercles have been equally affected. In one case (of which clinical notes are given) the optic paths were quite unaffected.

Cushing maintains that the adiposo-genital syndrome is a secondary manifestation, caused by the effect on the grey matter of the basal ganglia, of hormones secreted by a diseased hypophysis; and our cases seem to support this view.

The primary symptom, almost constant, is lowering of visual acuity. In the early stage of the disease this is generally unilateral, and not infrequently the patient discovers that the defect is in the temporal field. Ophthalmoscopic examination even when the defect is considerable reveals no visible change. This is easy of interpretation. A tumour in the chiasmal region usually causes serious interference with the conduction of visual impulses, becoming manifest early in the disease. Atrophy of the papilla results from secondary degeneration of the optic paths, and requires time to develop. When present, the atrophy of the disc may be more pronounced in the eye which retains more sight. Perimetric examination with coloured as well as white test objects should be carried out: we have seen a series of cases in which bitemporal achromatopsia, sectoral or complete, was the first signal 
of disease. Nevertheless bitemporal hemianopia is the classical sign in the early stage of chiasmal lesions, followed by amaurosis of the eye first attacked.

Defect of visual acuity is not dependent wholly on involvement successively of the crossed and uncrossed fibres in the chiasma. Central scotoma, due to interruption in the conductivity of the macular fibres is a calamitous feature in the evolution of chiasmal tumours. Occasionally homonymous hemianopia instead of the bitemporal variety is present $:$ it is a simple question of the direction of extension of the growth. If this affects chiefly the posterior angle of the chiasma bitemporal hemianopia will result ; an extension backwards on either side leads to involvement of the optic tract and homonymous hemianopia. Pleocytosis is not uncommon in the course of a tumour in the chiasmal region, and occasionally reaches a very high degree. This sign may confuse the diagnosis and lead to a suspicion of a specific infection such as encephalitis lethargica.

Secondary symptoms in chiasmal tumours are not infrequent and include the indications of high intracranial pressure; hence a diagnosis between a tumour in the chiasmal region and one elsewhere in the cranial cavity may become very difficult.

J. B. LAWFORD.

(2) Hamman, J. (Hamburg).-On the diagnosis and treatment of pituitary tumours. (Beitrag zur Diagnose und Therapie von Hypophysentumoren). Zeitschr. f. Augenheilk., Bd. LXVIII, p. 318, 1929.

(2) Hamman recalls the recent work on the pathology of pituitary tumours, and points out that the tumours associated with symptoms of acromegaly run a milder course than those which are not associated with acromegaly. These latter give rise more frequently to visual disturbances and other symptoms due to local pressure, and this is to be explained by the fact that this group is generally malignant. Papilloedema is uncommon in any type, and when present to any extent should give rise to the suspicion that the tumour is one growing from the infundibulum, and in its growth is compressing the base of the third ventricle.

The author records the results obtained in Behr's clinic in ten cases of pituitary tumours treated by radiation. In eight out of the ten cases X-rays only were used; in the two remaining radium emanations were also employed. The ten cases were all of the type in which symptoms of acromegaly were absent, and in all except one case failing vision was one of the chief symptoms; and defects in the field were present in eight out of the ten. On the whole the results of treatment were satisfactory. In no case did the treatment cause damage and in six cases there was a definite 
improvement, which could not have been obtained by any other means. In one case X-rays were applied subsequent to operative interference. In one case of doubtful pathology (? cyst, ? tuberculoma) the general condition improved but the eye condition was unaffected. One case which did not respond to treatment ultimately came to post-mortem examination, when it was found that the pituitary tumour was one of a series of metastases from hypernephroma.

Early diagnosis and more accurate diagnosis of the nature of pituitary tumours are essential in the treatment. In Hamman's opinion radiation gives the best results in cases where the X-ray examination does not show any bone destruction. Where bone destruction is present, and especially if the sight is threatened, operative interference is still indicated; but even in these cases a subsequent course of X-ray treatment should be given. For even if $\mathrm{X}$-rays do not destroy the tumour they help to form a fibrous wall around it and thus encapsulate it.

ARNold Sorsby.

(3) Magnus, Hans (Göttingen). - Roentgen-radiations of tumours of the hypophysis, together with remarks on hemianopic pupillary inaction. (Röntgenbestrahlungen der Hypophysentumoren, nebst Bemerkungen ueber hemianopische Pupillenstarre.) Arch.f. Ophthal., Vol. CXXI., p. 225.

(3) Magnus gives a short analysis of 34 cases of pituitary tumours treated in the Göttingen Eye Clinic since 1919. Twentytwo of these were treated with Roentgen radiations with improvement of vision in 50 per cent.; the best results were obtained in the cases of acromegaly. In none of the patients was any appreciable improvement in the general condition noted. In three cases he observed a symptom, not recorded in the literature but for which he offers no explanation, viz., a temporal pupillary inaction in the one amaurotic eye with absence of this in the other seeing eye.

Thos. SNowball.

(4) Coppez, Henri (Brussels).-The mechanism of lesions of the chiasma in fractures of the skull. (Le mécanisme des lésions du chiasma dans les fractures du crane). Arch. d'Ophtal., December, 1929.

(4) Coppez has had a recent opportunity of observing three cases of fracture of the skull in which the chiasmal syndrome was present. He publishes them partly because such examples are uncommon and partly because they serve to elucidate some doubtful points in the pathogenesis of these lesions and led him to undertake some experimental research. 
Case 1. Male, aged 21 years, found unconscious beside his motor-cycle. Comatose 12 hours. Injury chiefly to right side of face. Recovery with visual defect and some amnesia. Coppez examined him later and found bi-temporal hemianopia, macular regions intact with normal central vision. Radiographic examination : fracture of frontal bone (R) with slight depression; fracture of nasal septum in posterior part. Sella turcica, optic foramen and petrous bone normal.

Case 2. Male, aged 36 years. Fall down a staircase. Fracture with depression of left frontal eminence, penetration of splinters into dura mater and brain. Track of fracture found on surgical examination extending towards base of skull. Incomplete coma. A year later Coppez examined him and noted : R.V. $=0.8$, O.D. pale, pupil reactions normal. Field contracted concentrically, but with large temporal loss. L.V. $=0 \cdot 1, \mathrm{O} . \mathrm{D}$. atrophic, pupil reactions impaired, field concentrically contracted. Diagnosis, lesion of the left optic nerve, extending to the left border of the chiasma, where are grouped the fibres subserving the panoramic field of the right eye.

Case 3. Male, aged 9 years, knocked over by a motor car. Unconscious; wound extending from the outer angle of left eye to the left ear. Avulsion of the left eyeball. On recovery of consciousness $\mathrm{V} .=$ p.l. Next day it was possible to determine a large gap in the temporal field of the right eye. Fundus normal. Gradual recovery of vision so that 6 months later central acuity was normal, O.D. slightly pale, a crescentic loss of field on the temporal side, " representing the monocular panoramic field of the right eye." Radiographic examination revealed fracture of the outer wall of the orbit with depression of bone into the orbital cavity. The sphenoidal fissure and the optic foramen also showed signs of fracture.

In the literature of this subject it is noteworthy that there is no record of an autopsy demonstrative of the mechanism of the lesions of the chiasma. Liebrecht was present at an autopsy made two years after a fracture of the skull : bi-temporal hemianopia had resulted from the injury. The track of a fracture passing along the ethmoid and small wing of the sphenoid and terminating just in front of the right optic canal was found. There was no evidence of fracture in the immediate vicinity of the chiasma. Unfortunately the optic nerves and chiasma were lost.

The frequent escape of the chiasma in fractures of the skull is due to its position; it is so placed that it is not easily reached by the course of a fracture or by splinters of bone. Even if the line of fracture passes across the body of the sphenoid and the optic groove it may not touch the chiasma. The vulnerable points of the chiasma, in the author's opinion, are constituted by the optic 
nerves in their course from the anterior angles of the chiasma to the optic canals, to which they are firmly attached. The tissue of the chiasma is more fragile than that of the optic nerves, as can be demonstrated both clinically and anatomically.

The chiasmal syndrome is met with in three varieties of fracture :- -1 . Fracture of the fronto-sphenoidal arch, in which the fracture involves the vault of the orbit and the optic canal. It usually results from violent contusions in the frontal region, generally accompanied by loss of consciousness and commotio cerebri. As a rule the optic nerve is destroyed at the level of the optic canal and one-sided blindness results. Occasionally, however, bi-temporal hemianopia has been noted. Liebrecht suggested that the mechanism of the damage to the optic paths is as follows : In severe blows on the forehead the transverse diameter of the cranial cavity is suddenly increased, the ethmoid is flattened anteroposteriorly (and may be fractured). The two optic canals violently separated from one another drag with them the optic nerves which in turn drag on the chiasma. If the force is sufficient the chiasma is torn sagitally and bi-temporal hemianopia ensues. This is complete if the chiasma is torn through, but if some crossed fibres escape small islands of vision may be retained in the temporal field. Monocular vision as a rule is not lost.

Coppez has reproduced these lesions experimentally on the cadaver less than six hours after death. His results and the illustrations thereof should be studied in his paper: they are of, great interest. His general conclusions are that the lesions of the chiasma are induced by the agency of the optic nerves, and not by that of the bones, dura mater or haemorrhage.

2. Antero-lateral fracture with depression of the external orbital wall, extending to the sphenoidal fissure and the optic canal. The contents of the orbit are expelled by pressure, avulsion of the globe occurs and the optic nerve is dragged with it. The nerve ruptures at the anterior orifice of the optic canal, but if the latter is involved in the fracture the nerve is torn away at the level of the chiasma. The few recorded cases of this kind show that when avulsion of the eyeball occurs, complicated by the chiasmal syndrome, the apex of the orbit and the optic canal have always been involved. In Coppez's Case 3, the loss in the visual field corresponded exactly to the " panoramic visual field." Wilbrand has shown that at the level of the chiasma the panoramic optic fibres are collected (after their crossing) in the outer and posterior part of the chiasma. By experiment Coppez has confirmed the evidence.

3. Fracture of the petro-occipital arch spreading to the petrosphenoidal. Fractures in this area follow a line parallel to the 
axis of the petrous bone; they may involve the sella from behind forwards or from without inwards. We may surmise that the optic nerve is abruptly and violently pulled by the separation of the bones, this separation being directly in the course of the nerve from the commissure to the optic canal. The clinical features in this group of cases support this surmise. There is not only bi-temporal hemianopia but central vision is damaged, visual acuity is lowered and the ophthalmoscope shows optic nerve atrophy.

In this group experiment failed to produce the lesions thought to underlie the clinical symptoms: hence the possibility of some factors which are not disclosed by experiment, cannot be excluded.

In the opinion of the author clinical and experimental observations indicate that the lesions of the chiasma and the consequent chiasmal syndrome in these cases of fracture are the result of severe traction by the optic nerve causing laceration of the commissure.

J. B. LAWFORD.

\section{II. -EYE INJURIES}

(I) Fisher, J. Herbert (London).-Perforating wounds of the eyeball. (The Hunterian Lecture delivered before the Royal College of Surgeons of England, on February 5, 1930). Lancet, April 12, 1930.

(1) Fisher's Hunterian Lecture, while delivered before the Royal College of Surgeons as a whole, appears to be specially addressed to those women and men (in that order) who contemplate the practice of ophthalmology. The subject chosen is the very important and difficult one of perforating wounds of the eyeball, a subiect which almost more than any other in ophthalmic practice has its pitfalls for those without long experience. The author takes seven cases in detail and studies their points fully, giving in each case the reasons for the procedures adopted. There is one thing that stands out prominently in this lecture and that is the value in Fisher's opinion of the scleral route for the extraction of foreign bodies behind the lens, provided correct localisation is obtained beforehand. Into this question of localisation the author enters fully, pointing out that at Moorfields prior to 1916 proper radiographic charting was not employed. "The figures supplied by the radiologist were the three measurements of the site of the foreign body, vertically, horizontally, and posteriorly, in reference to the central point of the cornea. That such exact 
practitioners as ophthalmic surgeons should have been in the habit of using in combination only two at a time of the three measurements given is hardly to be believed." Fisher then quotes, from his own article in the Ophthalmı: Review for 1916, his criticism of this state of affairs and shows how since 1916 the charts have been altered so that much more exact localisation can be obtained from the figures supplied by the radiologist. The reviewer purposely refrains from the endeavour to synopsise the cases. Every ophthalmic surgeon will be glad to study them in full, but the nature of the cases may be stated here. Case I. Explosion of chemicals. Case II. Explosion of percussion cap. Case III. Bursting of chemical glass flask. Case IV. Bursting of electric light bulb. Case V. Fragment of steel from a hammer. Case VI. Intra-ocular changes produced by a non-penetrating blow with a pellet of shot. The point in this case is that had the old method of localisation been employed, the inference would have been that the pellet was intra-ocular. Case VII. Traumatic tumour of the iris.

A few words follow regarding siderosis. Referring to the discussion as to the source of the pigment, Fisher concludes that the iron containing pigment is derived not from the blood but from the retained foreign body.

Finally, Fisher's description of his operation by the scleral route in Case V. may be of value to those who do not habitually employ this procedure. It is understood that the position of the foreign body has been ascertained. "I turned back a flap of ocular conjunctiva and inserted in the superficial layers of the exposed sclerotic two fine silk sutures, parallel to one another and about two millimetres apart, near to the equator of the eyeball at the temporal edge of the superior rectus muscle; these stitches were left long and untied. One stitch I held taut myself, my assistant doing the same with the other while, with a Graefe's cataract knife, I cut slowly and carefully through the sclera between the two stitches until the pigmented choroid was visible. The knife was then plunged through choroid and retina, the sterilised point of the hand electro-magnet was applied to the wound, and the chip of steel was at once withdrawn. Taking at once each of the stitches into my hands I tied them together and cut them short; the conjunctival flap was then replaced and secured with fine silk stitches. I am still an advocate of the scleral incision for the removal of intra-ocular foreign bodies located behind the lens. We have now at our disposal very powerful electro-magnets of the Haab and Mellinger type. Even when the most precise steps are followed in using these powerful tractors, I consider it impossible by any means to be sure that serious damage to lens, iris or ciliary body 
will not be done in pulling metallic fragments forwards into the anterior chamber from the deeper parts of the eye. I have found it quite possible to use the Mellinger pattern by the scleral route."

ERNEST ThOMSON.

(2) Begle, Howell L. (Detroit).-Perforating injuries of the eye by small steel fragments. Amer. Jl. of Ophthal., December, 1929.

(2) Begle has been in practice for 25 years in the heart of the motor manufacturing district of Michigan, and so has had unusual opportunities for observing and treating injuries to the eye by metallic foreign bodies. He has seen altogether 250 cases of penetrating wounds of the eye. His present paper is limited to the injuries produced by small fragments. If the perforation of the cornea is near the limbus, the foreign body may pass through the zonule and leave the lens uninjured. In only one out of ten cases of this kind did the lens show any change and in this instance there was only a limited flame-shaped opacity near the equator. Eight patients obtained useful vision after removal of the fragment by the posterior route; of the remaining two, one had the fragment embedded in the retina and developed a detachment, while the other was an old injury with siderosis and degenerative changes. In all cases there was a hole or slit in the iris, and although there was some blood in the vitreous, the foreign body was usually visible, though twice it was located behind the ciliary body where, of course, it was out of reach of the ophthalmoscope. If the foreign body is of larger size, the zonular region is not of sufficient width to prevent injury to the lens and ciliary body, so that severe haemorrhage and cataract frequently result. There were three cases with a steel fragment in the lens, in each of which the latter remained clear after extraction of the fragment, except for the scar in the anterior capsule. In describing these cases the author gives a useful detail of technique; namely to allow the fragment to remain in the anterior chamber for 24 hours after its extraction from the lens, so as to permit the rent in the capsule to close while the chamber is full. The fragment is then removed by the magnet through a tangential incision in the cornea. The weight of the foreign body in these cases was about half a milligramme. If the case is not seen until some time after the original injury, e.g., five or six weeks, the capsular wound will have completely healed and it may not be possible to draw the fragment of metal through it. In such cases a cystitome has been used to make a small incision through the capsule. The fragment is then removed through this incision and there may be no further development of lens opacity. 
An alternative procedure, when the fragment is sharp, is to make and break the current repeatedly, and thus induce it to perforate the capsule. The author has had fourteen cases of small foreign bodies traversing the entire thickness of the lens, of which seven recovered useful vision, the opacity being limited to a punctate one at the point of entry in the anterior capsule and a disc-shaped opacity in the posterior capsule at the point of exit.

$$
\text { F. A. W.-N. }
$$

\section{(3) Nutting, R. J.-Industrial corneal injuries. California and} Western Medicine, May, 1929.

(3) Nutting has collected and analysed 1,400 consecutive cases of corneal injury. Of these 1,382 were non-penetrating and of these $64 \%$ were due to iron or rust scales in various forms. Cases coming direct to hospital required an average treatment of 1.53 days' duration, while referred cases needed attention for an average of 3.89 days. The various forms of treatment adopted are given and the value of neutral ammonium tartrate in lime burns is pointed out.

He concludes by urging a wider use of protective goggles for workers and suggests that employers should placard the shops with notices cautioning workmen against trying to remove objects from injured eyes of their fellows, as such well meant efforts often lengthen the time needed for recovery.

\section{R. C. Davenport.}

(4) Cridland, B. - Minor injuries to the eye in industry. Brit. Med. Jl., October 5, 1929.

(4) Cridland first quotes figures showing the incidence of blindness due to accident and the toll of such accidents in industry. This is reflected in the figure he gives-53\% of all out-patients at his own hospital in 1928-for accidents in hospital practice.

With others he investigated a series of cases of minor injury in regard to the lesion, the question of protection to the eyes at work, and further, the time lost to industry as a sequel to the accidents. Other figures are quoted and in spite of the difficulties involved, it is urged that the prevention of minor injuries to the eyes could be assured by a combination of the practice of three principles-mechanical guarding of the eyes, education of workmen and foremen and, ideally, engineering revision with a view to the avoidance of flying particles.

R. C. Davenport. 
(5) Yoshimoto, Ryottin (Japan). - On experimental acid and alkali burns of the eye. (Ueber experimentelle Säure-und Lageverätzungen der Augen). Arch. f. Augenheilk., Bd. XCIX, May, 1928.

(5) These experiments were carried out in the Leipzig University Eye Clinic on full grown rabbits' eyes. Yoshimoto summarises the research as follows:-

1. According to the severity of the burn the alkalies and acids employed could be graded as caustic soda, hydrochloric, sulphuric, nitric, acetic acids and ammonia.

2. Burns from caustic soda in concentration $\mathrm{n} / 2$ and upwards, hydrochloric or sulphuric acids in $\mathrm{n} / 1$ and upwards, nitric acid in $2 \mathrm{n}$ and upwards, acetic acid in $3 \mathrm{n}$ lead to phthisis bulbi. Burns from $3 \mathrm{n}$ ammonia result in the formation of leucoma corneae.

3. If, not later than 5 minutes after a caustic soda burn, thorough irrigation with slightly acidulated water or $1 \%$ tannin solution is carried out, leucoma or phthisis bulbi could be prevented, provided the concentration of the alkali is no higher than $\mathrm{n} / 2$.

4. Provided the concentration of the acid is no higher than $n / 1$, thorough washing out with slightly alkaline water or $1 \%$ tannin solution, within 5 minutes after hydrochloric, sulphuric and nitric acid burns, prevents leucoma and phthisis bulbi. Similar treatment gives a better prognosis in acetic acid burns.

5. Anatomical examination showed that alkali burns are more intensive and extensive, and that repair sets in very much later than after acid burns.

D. V. GIRI.

(6) Jess, A. (Giesen). - The disappearance of the manifestations of copper in the eye. (Das Verschwinden des Verkupferungserscheinungen des Auges.) Zeitschr. $f$. Augenheilk., Vol. LXIX, p. 59, 1929.

(6) Owing to the fact that the ammunition used by Germany during the war contained but little copper, chalcosis as seen in Germany tends to run a milder course than in other countries, for most cases of chalcosis are the result of accidents in which ammunition is involved. Jess records his experience of 13 cases, in 5 of which he observed the disappearance of fully established manifestations of copper.

When chalcosis does not run a rapid course ending in abscess formation, the commonest changes are to be observed in the lens, and these have a typical appearance, giving rise to a cataract of 
a shape aptly described as a sunflower type-a cataract with a solid centre and radiating peripheral " petals." It is greenish in colour, and with the slit lamp the lesion is seen to be subcapsular, the rest of the lens being clear. The subcapsular site of the lesion was shown histologically by the author in a case of his that ended in enucleation; no copper was seen in the epithelium, though Vogt holds that the epithelium is the primary site of the copper impregnation.

An interesting feature of the peripheral " petals " is seen in the fact that when the pupil is kept dilated the opacity spreads to the whole area of the lens; these "petals" are, therefore, somehow, connected with the iris : they probably represent the permanent folds on the posterior surface of that structure.

Apart from lens changes chalcosis may also manifest itself by a greenish tinge in the cornea owing to the deposition of the copper salts between the endothelium and Descemet's membrane; a pale green colour may also be seen in the iris, zonula and the vitreous. In the fundus a golden-sand appearance has been observed at the macula.

In the 13 cases observed by the author, the typical copper cataract was seen in 11 cases; in 10 the cornea was involved; coloration of the iris was noted in 8 cases, but the greenish tinge is difficult to be sure of in dark irides. In the vitreous, changes were seen nine times, and in the retina eight. Of these 13 eyes (all cases were unilateral) four ended in enucleation, in three of these on account of iridocyclitis and in one on account of absolute glaucoma. Of the nine remaining cases two were lost sight of, leaving seven, of which five showed the complete disappearance of copper cataract which had been observed in them at earlier stages. There is apparently no definite time limit during which the manifestations of chalcosis may clear up ; in these five cases the lenses became clear, $6,8,10,12$ and 16 years respectively after the original injury. It would also appear that there is no time limit when the copper cataract may first show itself : it may be a matter of months or years : apparently it comes on earlier in younger subjects. In one of the five cases vision was completely restored after the clearing up of the lens changes and the other manifestations of chalcosis; but generally there is some permanent impairment owing to traumatic changes induced by the foreign body. In one case the lens became clear four years after a particle of copper had been removed from the anterior chamber.

The author concludes that unlike siderosis, there is in chalcosis no irreversible combination with the cellular protein, and that the tendency for chalcosis is to disappear. Provided iridocyclitis and glaucoma do not supervene there is a good prospect of restoration of useful vision. On the basis of his experience Jess 
advises non-interference, unless the copper be easily removed or forces the surgeon's hand on account of recurrent iridocyclitis or rising tension. A waiting policy is all the more justified as there seems to be but little danger of sympathetic ophthalmitis in chalcosis.

ArNold SORSBY.

\section{BOOK NOTICES}

Stereoskopischer Atlas der äussern Erkrankungen des Auges. By KARL WeSSELY (München). A second series of ten stereophotographs in colour ( $2 \frac{1}{2}$ in. by 2 in. on cards, 7 in. by 4 in.). München: J. F. Bergmann. Price, R.M. 12.00.

A first series of ten stereo-photographs in colours was reviewed on page 138 of the present volume of this Journal. Of the ten photographs in this second series three deal with such gross lesions as cicatricial entropion due to advanced lupus, abscess formation in acute dacryocystitis and dislocation of the whole of the eyeball. These three conditions are successfully illustrated, but it must be confessed that the photographs dealing with finer lesions, such as for instance the one depicting a splinter of iron in the iris, leave much to be desired, as far as stereoscopic representation goes. Of the twenty photographs thus far published, the most successful are undoubtedly those dealing with gross and essentially cutaneous lesions. Nothing approaching the same standard of excellency is to be found in the photographs of the purely ocular affections. The technical difficulties associated with colour in stereo-photography have apparently not been overcome altogether.

Morax et Petit. Le Trachome. Editeur: J an Morax. Paris. 1929.

Since Morax published his clinical researches on trachoma in Egypt in Ann. d' Ocul. in 1901 and 1902 he has been looked on as one of the greatest authorities on this disease. As the founder of, and principal contributor to, La Revue Internationale du Trachome, a quarterly journal, he has retained the study of trachoma in all its aspects as one of the professional interests of his life.

A book by him on trachoma has been awaited eagerly, and now 